\title{
Orange/Red Fluorescence of Active Caries by Retrospective Quantitative Light-Induced Fluorescence Image Analysis
}

\author{
Grace Felix Gomez $^{\mathrm{a}}$ George J. Eckert ${ }^{\mathrm{b}, \mathrm{c}}$ Andrea Ferreira Zandona ${ }^{\mathrm{d}}$ \\ ${ }^{a}$ Department of Oral Biology, Indiana University School of Dentistry, ${ }^{b}$ Department of Biostatistics, \\ Indiana University School of Medicine, and ' Department of Biostatistics, Richard M. Fairbanks School of \\ Public Health, Indianapolis, Ind., and d Department of Operative Dentistry, School of Dentistry, \\ The University of North Carolina at Chapel Hill, Chapel Hill, N.C., USA
}

\section{Key Words}

Biofilm · Caries · Clinical study · Digital image analysis · Red fluorescence

\begin{abstract}
This retrospective clinical study determined the association of caries activity and orange/red fluorescence on quantitative light-induced fluorescence (QLF) images of surfaces that progressed to cavitation, as determined by clinical visual examination. A random sample of QLF images from 565 children (5-13 years) previously enrolled in a longitudinal study was selected. Buccal, lingual and occlusal surface images obtained after professional brushing at baseline and every 4 months over a 4-year period were analyzed for red fluorescence. Surfaces that progressed $(n=224)$ to cavitation according to the International Caries Detection and Assessment System (ICDAS 0/1/2/3/4 to 5/6 or filling), and surfaces that did not progress $(n=486)$ were included. QA2 image analysis software outputs the percentage increase of the $\mathrm{red} / \mathrm{green}$ components as $\Delta \mathrm{R}$ and area of $\Delta \mathrm{R}$ (area $\Delta \mathrm{R}$ ) at different thresholds. Mixed-model ANOVA was used to compare progressive and nonprogressive surfaces to account for correlations of red fluorescence $(\Delta R$ and area $\Delta R)$ between surfaces within a subject. The first analysis used the first observation for each surface or the first available visit if the sur-
\end{abstract}

face was unerupted (baseline), while the second analysis used the last observation prior to cavitation for surfaces that progressed and the last observation for surfaces that did not progress (final). There was a significant $(p<0.05)$ association between red fluorescence and progression to cavitation at thresholds $\Delta \mathrm{R} 0, \Delta \mathrm{R} 10, \Delta \mathrm{R} 20, \Delta \mathrm{R} 60, \Delta \mathrm{R} 70, \Delta \mathrm{R} 80, \Delta \mathrm{R} 90$ and $\Delta R$ max at baseline and for $\Delta R 0$ and $\Delta R 10$ at the final observation. Quantification of orange/red fluorescence may help to identify lesions that progress to cavitation. Future studies identifying microbiological factors causing orange/ red fluorescence and its caries activity are indicated.

(c) 2016 S. Karger AG, Basel

The optical phenomenon of tooth autofluorescing on illumination with ultraviolet light was first observed by Hans Stubel [Stubel, 1911; Buchalla, 2005]. This property has been applied in caries detection devices as a means of detecting incipient carious lesions [Benedict, 1928; Alfano and Yao, 1981; Bjelkhagen et al., 1982; Sundstrom et al., 1985; de Josselin de Jong et al., 1995; Zandona and Zero, 2006]. The concept held previously was that carious enamel lesions do not fluoresce [Benedict, 1928], and deep dentinal caries appeared dark brown, orange brown and pinkish orange when caries was debrided. It was shown later that carious lesions fluoresce in the red region

\section{KARGER}

E-Mail karger@karger.com

www.karger.com/cre
(C) 2016 S. Karger AG, Basel

0008-6568/16/0503-0295\$39.50/0
Andrea Ferreira Zandona, DDS, MSD, PhD

Department of Operative Dentistry, School of Dentistry

The University of North Carolina at Chapel Hill

436 Brauer Hall, Room 447, Chapel Hill, NC 27599-7450 (USA)

E-Mail azandona@email.unc.edu 
Table 1. Description of QLF parameter terminologies

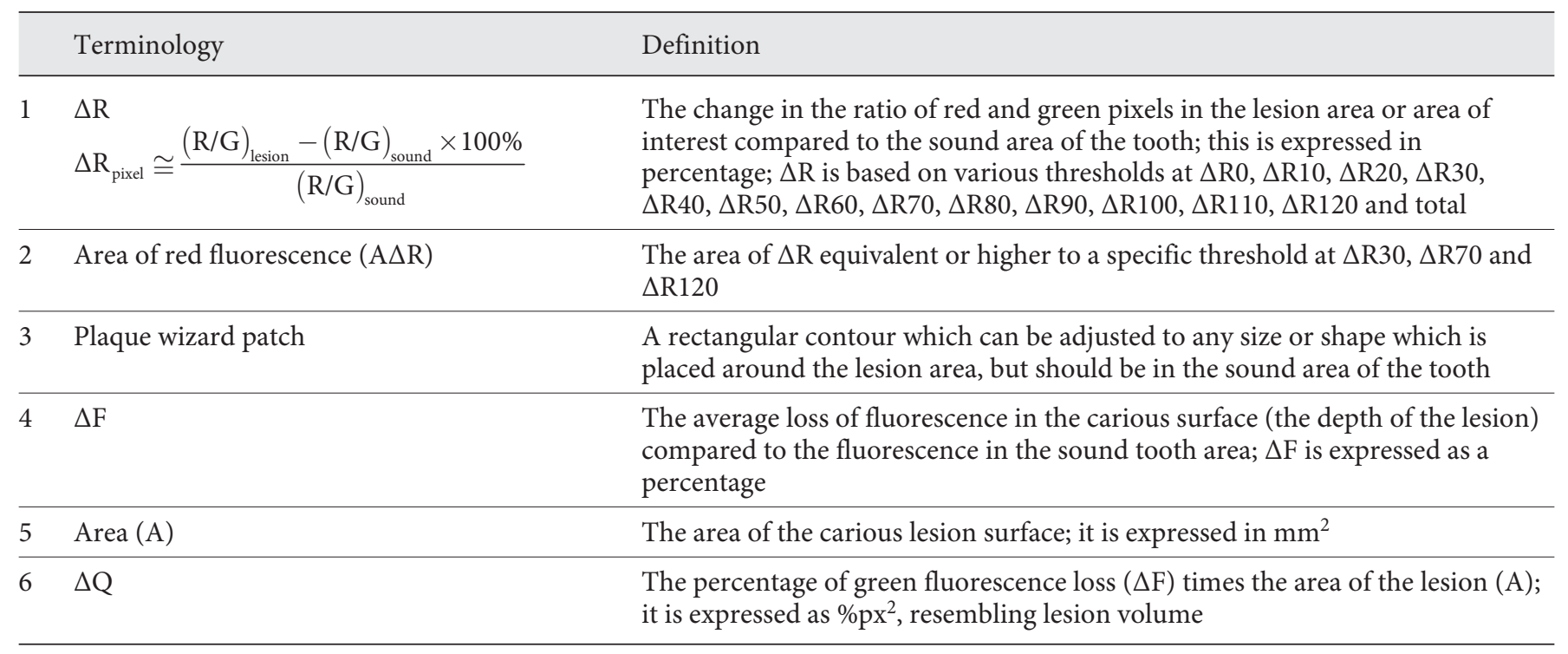

of the spectrum [König et al., 1999; Buchalla, 2005; Slimani et al., 2014]. Several studies have demonstrated heterogeneous emission spectral bands in the carious region compared to the sound surface of the tooth [Buchalla et al., 2004a, b; Buchalla, 2005; Zezell et al., 2007]. The longstanding question is whether the chromogenic autofluorescence originates from the tooth structure or from bacteria, or whether both contribute to this autofluorescence. It has been shown that macromolecular porphyrin might be responsible for the autofluorescence [Buchalla et al., 2008].

Quantitative light-induced fluorescence (QLF-CLIN; Inspektor Research Systems, Amsterdam, The Netherlands) employs the optical property of fluorescence for caries detection [Waller et al, 2012]. Inspektor ${ }^{\mathrm{TM}}$ QLF Pro employs an intraoral camera that illuminates the tooth with light in the violet-blue wavelength $(290-450 \mathrm{~nm})$ and captures fluorescence above $520 \mathrm{~nm}$ by a high-pass filter. Under these conditions, the sound areas of the tooth fluoresce green; however, an orange/red fluorescence can also be observed on these images. A new device, the QLF-D Biluminator ${ }^{\mathrm{TM}}$ 2, has been introduced using a single lens reflex camera with blue and white LED lights with a peak wavelength of $405 \pm 20 \mathrm{~nm}$ (violet). White LEDs are used for standard white light images, while the blue LEDs provide excitation light for fluorescent imaging. The filter in this device allows the images of sound tooth surfaces to have a whitish appearance instead of green, while demineralized areas look darker, and bacterially infected areas show a bright red fluorescence. The orange/red fluorescence has been assumed to originate from metabolic byproducts of oral bacteria within the dental biofilm called porphyrins [König et al., 1998; van der Veen et al., 2006; Volgenant et al., 2013]. In vitro oral biofilm studies have shown a correlation between the red fluorescence captured through the QLF-D Biluminator with caries risk severity [Lee et al., 2013; Kim et al., 2014]. A proprietary software (Inspektor Pro System) allows analysis of the images reporting three parameters: the average loss of fluorescence denoting lesion depth $(\Delta \mathrm{F}, \%)$, lesion area $\left(\mathrm{A}, \mathrm{mm}^{2}\right)$ and lesion volume $\left(\Delta \mathrm{Q}, \% \mathrm{px}^{2}\right)$. In the QLF-D Biluminatorthe ratio of red to green fluorescence in percentage $(\Delta \mathrm{R}, \%)$ and the area of red fluorescence $(\mathrm{A} \Delta \mathrm{R})$ are reported. Red or orange fluorescence $(\Delta R)$ is represented as the percentage ratio increase of the red to green components in comparison to sound surface. The red fluorescence area $\left(\mathrm{A} \Delta \mathrm{R}, \mathrm{px}^{2}\right)$ is equal or higher than a specific threshold of $\Delta \mathrm{R}$ (table 1 ). Red or orange fluorescence is believed to emanate from the excitation of fluorophores from bacterial byproducts on illumination through blue-violet light [van der Veen et al., 2003]. A preliminary in vitro study [Alammari et al., 2010] showed the relationship between the $\Delta \mathrm{R}$ and $\Delta \mathrm{F}$ to ICDAS and histology scores, requiring clinical validation.

The relationship between the QLF parameters $(\Delta \mathrm{F}, \Delta \mathrm{A}$ and $\Delta \mathrm{Q}$ ), the visual appearance of the lesions and its clinical behavior longitudinally has been demonstrated previously by our group [Ferreira Zandona et al., 2003, 2010]. During the capture and analysis of these clinical images the red fluorescence observed by others [Coulthwaite et 
al., 2006; Bittar et al., 2014] was also seen on the images captured in these longitudinal studies. Thus, the objective of this study was to determine whether there is an association between the intrinsic orange/red fluorescence seen in QLF images of surfaces that progressed to cavitation over a period of time compared to surfaces with noncavitated lesions that did not progress to cavitation, as determined by clinical visual examination using the criteria of the International Caries Detection and Assessment System (ICDAS).

\section{Study Participants and Methods}

This is a retrospective study based on the images captured during a longitudinal study previously published [Ferreira Zandona et al., 2013]. Detailed information of participant selection, sample size calculation, examinations conducted and the analysis of fluorescence in QLF images have previously been published [Ferreira Zandona et al., 2010; Fontana et al., 2011; Ferreira Zandona et al., 2012,2013 ]. In summary, in January 2007, children $(n=565)$ aged 5-13 years from kindergarten to 9th grade enrolled in public schools from the area of Aguas Buenas, Puerto Rico, were recruited into the study. Consent and assent if the child was over 7 years old was obtained. The study was approved by the Institutional Review Board (IRB) of Indiana University (IU-IRB 0608-15) and the University of Puerto Rico (UPR-IRB A1340107). The inclusion criteria included having at least 1 permanent molar with at least 1 unrestored surface, no medical problems and tolerance for the examinations performed.

\section{Comprehensive Oral Examinations}

The children underwent examinations at baseline and at 8,12 , $18,24,28,32,36,40,44$ and 48 months. It is important to note that the examinations were conducted after a professional toothbrushing; thus most, if not all, the biofilm was removed from the surfaces of interest. A visual examination based on ICDAS, which ranges from 0 to 6 [Pitts, 2004], was performed at each time point as well as an examination of the teeth with QLF. Fluorescent images of occlusal and buccal surfaces of all permanent molars and lingual surfaces of upper molars were obtained at each examination. At the end of the 4-year study fluorescent images of surfaces that progressed to cavitation (ICDAS $0 / 1 / 2 / 3 / 4$ to $5 / 6$ or filling) and a random sample of images of surfaces that did not progress to cavitation were analyzed longitudinally using proprietary software (QLF $2.00 \mathrm{~g}$ ).

\section{Orange/Red Fluorescence Analysis}

The same images previously analyzed were analyzed for orange/red fluorescence by red fluorescence analysis (QA2 software; Inspektor Research Systems). Surfaces $(\mathrm{n}=224)$ that progressed to cavitation according to ICDAS (ICDAS $0 / 1 / 2 / 3 / 4$ to $5 / 6$ or filling) and surfaces that did not progress $(n=486)$ were included. [Ferreira Zandona et al., 2013]. The plaque wizard patch of QA2 of the QLF-D Biluminator was used to analyze the images obtained from the Inspektor QLF Pro. For red fluorescence analysis the plaque wizard was applied on the same area of the carious lesion surface which had been analyzed previously to monitor the progression of

Orange/Red Fluorescence of Active

Carious Lesions caries. It was applied on the last visit for sites that did not progress, or if there was a filling or a cavitation with ICDAS 5 or 6 , it was applied on the visit prior to the filling/cavitation. A similar plaque wizard was applied at all other visits including the first observation, which was at baseline, or the first visit after eruption if the tooth was unerupted at baseline. QA2 outputs the percentage increase of red to green components as $\Delta \mathrm{R}$ and area $\Delta \mathrm{R}$ at different thresholds. For intraexaminer reliability 60 surfaces were chosen randomly, including surfaces that progressed to cavitation and those that did not progress, for repeat analysis by a single, experienced examiner.

\section{Statistical Analysis}

Statistical analysis was performed with SAS 9.3 (SAS Institute Inc., Cary, N.C., USA). Mixed-model ANOVA was used to compare surfaces that progressed to cavitation and those that did not progress to account for correlations of red fluorescence $(\Delta \mathrm{R}$ and area $\Delta R$ ) between surfaces within a subject. The ranks of the data were used in the analysis because of nonnormal distributions. Two sets of analyses were performed. The first analysis used the first observation for each surface, which was at baseline or the first available visit if the surface was unerupted at baseline. The second analysis used the last observation before progression for surfaces that did progress to cavitation and the last observation for sites that did not progress. The area under the receiver operating curve (ROC) was calculated to determine the sensitivity and specificity of the threshold for the red fluorescence areas. Intraclass correlation coefficients were calculated to determine the level of intraexaminer repeatability.

\section{Results}

A statistically significant association $(\mathrm{p}<0.05)$ was seen between red fluorescence and surfaces that progressed to cavitation. Analysis of the initial or first observation of the tooth surfaces showed that sites that progressed to cavitation had a significantly higher percentage of red fluorescence for specific thresholds of $\Delta \mathrm{R} 0, \Delta \mathrm{R} 10$, $\Delta \mathrm{R} 20, \Delta \mathrm{R} 60, \Delta \mathrm{R} 70, \Delta \mathrm{R} 100, \Delta \mathrm{R} \max$ and total than sites that did not progress. The red fluorescence area (A $\Delta \mathrm{R}$ : the area at $\left.\Delta 70, \mathrm{px}^{2}\right)$ correlated with the percentage of red fluorescence observed in the first observation $(p=0.0191)$. The simple plaque score (SPS ${ }^{\mathrm{TM}}$ ) was borderline significant $(\mathrm{p}=0.0522)$ at the initial observation (table 2$)$.

At the first observation the mean value (and standard error, $\mathrm{SE})$ of red fluorescence values was 4,955 $(\mathrm{SE}=285)$ for surfaces that progressed to cavitation and 3,038 (SE = 166) for surfaces that did not progress. As shown in table 2 the sites that progressed to cavitation had significantly higher $\Delta \mathrm{R} 0, \Delta \mathrm{R} 10, \Delta \mathrm{R} 20, \Delta \mathrm{R} 60, \Delta \mathrm{R} 70, \Delta \mathrm{R} 100$, $\Delta R \max$ and total at the first observation than sites that did not progress. Even at the thresholds where there were no significant differences between the sites, there was a tendency for the sites that did not progress to have lower 
Table 2. Red fluorescence analysis of baseline and final observations of progressive and nonprogressive surfaces

\begin{tabular}{|c|c|c|c|c|c|c|}
\hline \multirow{2}{*}{$\begin{array}{l}\text { Red } \\
\text { fluorescence } \\
\text { parameter }\end{array}$} & \multicolumn{3}{|c|}{ First or baseline observation } & \multicolumn{3}{|c|}{ Final observation or last before progression } \\
\hline & $\begin{array}{l}\text { progressed to } \\
\text { cavitation } \\
(\mathrm{n}=224)\end{array}$ & $\begin{array}{l}\text { did not } \\
\text { progress } \\
(n=486)\end{array}$ & $\mathrm{p}$ value & $\begin{array}{l}\text { progressed to } \\
\text { cavitation } \\
(\mathrm{n}=224)\end{array}$ & $\begin{array}{l}\text { did not } \\
\text { progress } \\
(n=486)\end{array}$ & $\mathrm{p}$ value \\
\hline SPS & $0.045 \pm 0.021$ & $0.019 \pm 0.011$ & 0.0522 & $0.067 \pm 0.031$ & $0.029 \pm 0.009$ & 0.8458 \\
\hline Area $\Delta \mathrm{R} 30$ & $0.069 \pm 0.035$ & $0.039 \pm 0.019$ & 0.3948 & $0.142 \pm 0.086$ & $0.044 \pm 0.012$ & 0.4164 \\
\hline Area $\Delta \mathrm{R} 70$ & $0.016 \pm 0.010$ & $0.009 \pm 0.006$ & $0.0191^{*}$ & $0.022 \pm 0.017$ & $0.006 \pm 0.002$ & 0.4774 \\
\hline Area $\Delta \mathrm{R} 120$ & $0.000 \pm 0.000$ & $0.000 \pm 0.000$ & & $0.003 \pm 0.003$ & $0.000 \pm 0.000$ & 0.1905 \\
\hline$\Delta \mathrm{R} 0$ & $1,341 \pm 87$ & $752 \pm 48$ & $<0.0001^{*}$ & $1,449 \pm 96$ & $867 \pm 57$ & $<0.0001^{*}$ \\
\hline$\Delta \mathrm{R} 10$ & $43.00 \pm 5.06$ & $26.87 \pm 3.14$ & $0.0009^{*}$ & $51.17 \pm 6.27$ & $32.00 \pm 3.49$ & $<0.0001^{*}$ \\
\hline$\Delta \mathrm{R} 20$ & $5.96 \pm 1.45$ & $2.56 \pm 0.48$ & $0.0029^{*}$ & $9.55 \pm 2.70$ & $3.72 \pm 0.70$ & 0.3863 \\
\hline$\Delta \mathrm{R} 30$ & $2.37 \pm 0.83$ & $0.84 \pm 0.28$ & 0.3770 & $4.43 \pm 1.96$ & $1.46 \pm 0.44$ & 0.3603 \\
\hline$\Delta \mathrm{R} 40$ & $1.38 \pm 0.58$ & $0.49 \pm 0.25$ & 0.2837 & $2.76 \pm 1.63$ & $1.01 \pm 0.37$ & 0.2869 \\
\hline$\Delta \mathrm{R} 50$ & $0.95 \pm 0.44$ & $0.34 \pm 0.20$ & 0.0538 & $2.04 \pm 1.35$ & $0.62 \pm 0.27$ & 0.4398 \\
\hline$\Delta \mathrm{R} 60$ & $0.64 \pm 0.29$ & $0.23 \pm 0.13$ & $0.0167^{*}$ & $1.27 \pm 0.83$ & $0.37 \pm 0.18$ & 0.2233 \\
\hline$\Delta \mathrm{R} 70$ & $0.33 \pm 0.16$ & $0.12 \pm 0.07$ & $0.0191^{*}$ & $0.71 \pm 0.43$ & $0.21 \pm 0.12$ & 0.4749 \\
\hline$\Delta \mathrm{R} 80$ & $0.17 \pm 0.09$ & $0.04 \pm 0.03$ & 0.0511 & $0.42 \pm 0.25$ & $0.13 \pm 0.10$ & 0.7095 \\
\hline$\Delta \mathrm{R} 90$ & $0.07 \pm 0.05$ & $0.02 \pm 0.01$ & 0.1440 & $0.33 \pm 0.23$ & $0.09 \pm 0.08$ & 0.3285 \\
\hline$\Delta \mathrm{R} 100$ & $0.02 \pm 0.01$ & $0.00 \pm 0.00$ & $0.0373^{*}$ & $0.28 \pm 0.20$ & $0.07 \pm 0.07$ & 0.4253 \\
\hline$\Delta \mathrm{R} 110$ & $0.01 \pm 0.01$ & $0.00 \pm 0.00$ & 0.1416 & $0.22 \pm 0.17$ & $0.06 \pm 0.06$ & 0.1905 \\
\hline$\Delta \mathrm{R} 120$ & $0.00 \pm 0.00$ & $0.00 \pm 0.00$ & & $0.18 \pm 0.15$ & $0.04 \pm 0.04$ & 0.1905 \\
\hline$\Delta \mathrm{Rmax}$ & $44.08 \pm 1.43$ & $35.85 \pm 0.75$ & $<0.0001^{*}$ & $47.71 \pm 2.14$ & $40.51 \pm 1.05$ & $0.0002 *$ \\
\hline Total & $4,955 \pm 285$ & $3,038 \pm 166$ & $<0.0001^{*}$ & $5,113 \pm 287$ & $3,204 \pm 169$ & $<0.0001^{*}$ \\
\hline
\end{tabular}

Values are means \pm SE. ${ }^{*} \mathrm{p}<0.05$, statistically significant.

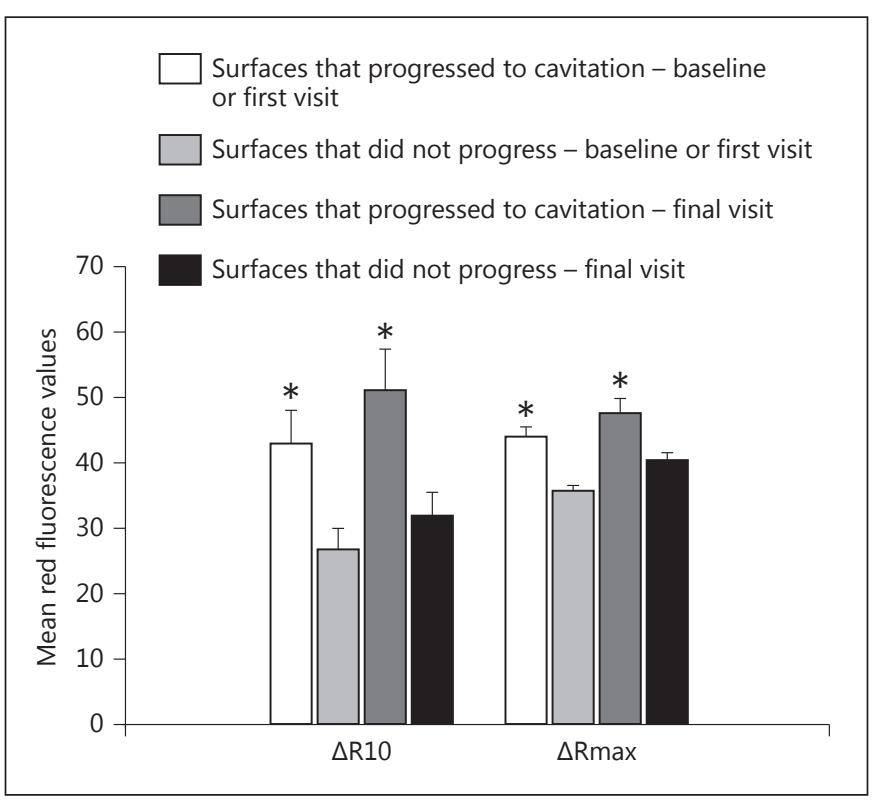

Fig. 1. Comparison of mean red fluorescence values at baseline or first visit and final visit of observation for surfaces that progressed to cavitation and those that did not progress at $\Delta \mathrm{Rmax}$ and $\Delta \mathrm{R} 10$. $* \mathrm{p}<0.05$. values than those that did progress to cavitation. The area at threshold $\Delta \mathrm{R} 70$ (area $\Delta \mathrm{R} 70)$ was significant for lesions that progressed to cavitation compared to the area at $\Delta \mathrm{R} 30$ and that at $\Delta \mathrm{R} 120$. Analysis of the final observation showed that sites that progressed to cavitation had significantly higher $\Delta \mathrm{R} 0, \Delta \mathrm{R} 10, \Delta \mathrm{Rmax}$ and total than sites that did not progress $(\mathrm{p}<0.0001)$. SPS scores were not significant at the last visit. The mean red fluorescence values at various thresholds during the final observation are included in table 2 . The areas of thresholds at $\Delta \mathrm{R} 30$, $\Delta \mathrm{R} 70$ and $\Delta \mathrm{R} 120$ were not significantly different.

Comparisons of red fluorescence values at $\Delta \mathrm{Rmax}$ and $\Delta \mathrm{R} 10$ during the first and final observation are illustrated in figure 1. For both the first and final observations there was a significant difference for $\Delta \mathrm{R} \max$ and $\Delta \mathrm{R} 10$ for surfaces that progressed to cavitation compared to nonprogressive surfaces. Carious surfaces that progressed to cavitation showed greater red fluorescence values during the final observation at $\Delta \mathrm{R} 10$. Surfaces that did not progress showed higher red fluorescence values at the first observation at $\Delta R \max$. Therefore, the threshold kept at maximum level of cutoff was greater compared to the red fluo- 
Fig. 2. Dot plot comparison of surfaces that progressed to cavitation and those that did not progress, with total values of red fluorescence during the first observation (a) and the final observation (b).

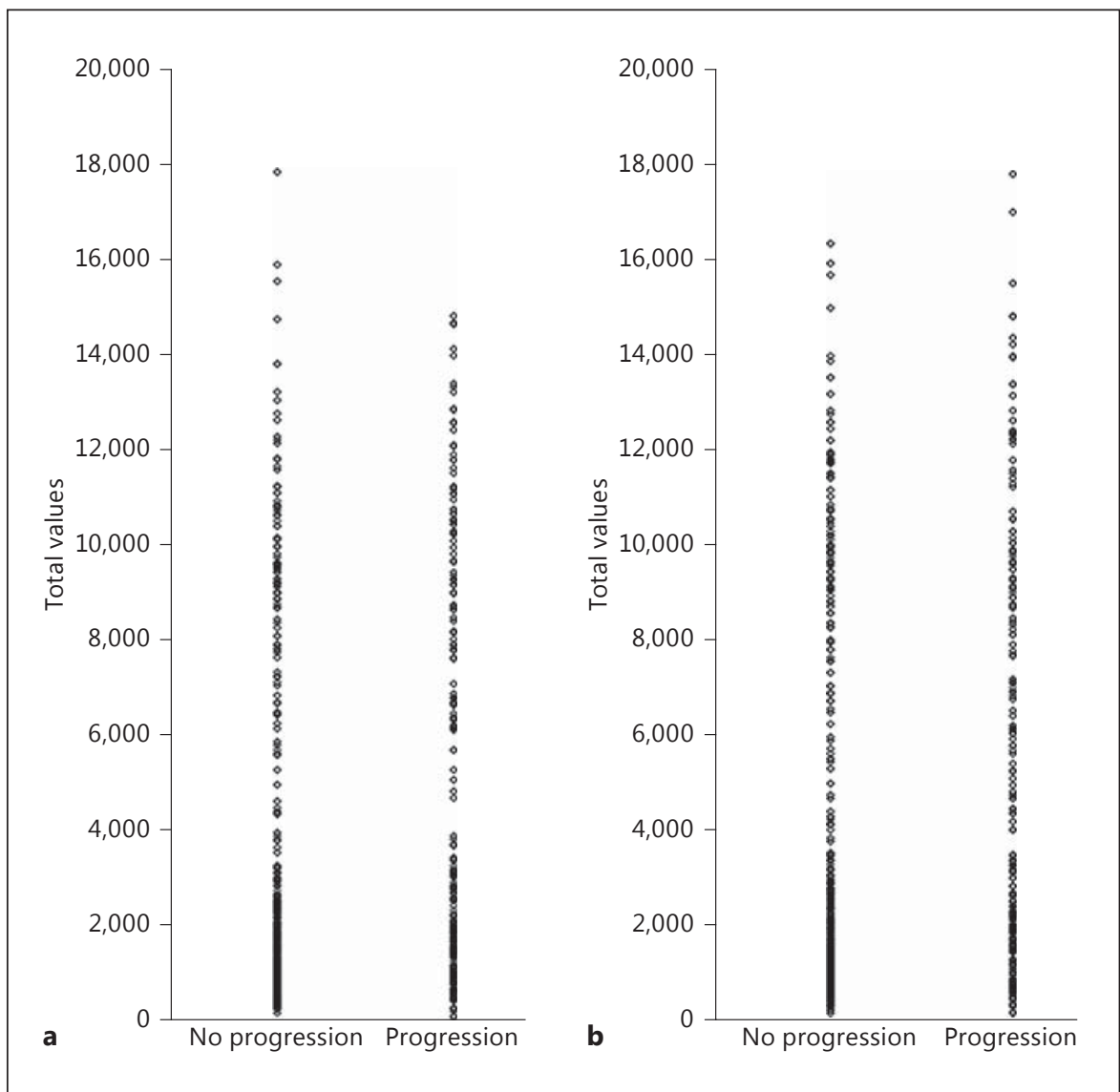

rescence value at $\Delta \mathrm{R} 10$. Red fluorescence is detected during the first observation with the maximum level of cutoff. Also, during the final observation of nonprogressive surfaces $\Delta R \max$ showed higher red fluorescence values.

Dot plots are shown comparing surfaces that progressed to cavitation and nonprogressive surfaces based on total values of red fluorescence during the baseline or first observation (fig. 2a) and during the final observation (fig. 2b). The surfaces that progressed to cavitation and nonprogressive surfaces were significantly different in both cases $(\mathrm{p}<0.0001)$.

The area under the ROC was calculated based on the continuous variables in the red fluorescence analysis data. Sensitivity and specificity were calculated based on the ROC. The ROC was plotted and was evaluated to determine the appropriate tradeoff between sensitivity and specificity. Figure 3 illustrates the ROC for the final observation (not progressed) or the last observation before progression to cavitation for the 'total' to predict progression. We can see from the curve that the best combination was a sensitivity of $65 \%$ with a specificity of $63 \%$.

Orange/Red Fluorescence of Active

Carious Lesions
The intraclass correlation coefficients indicated good repeatability for all red fluorescence measurements: 0.99 for $\Delta \mathrm{Rmax}, 0.97$ for red fluorescence total, 0.97 for $\Delta \mathrm{R} 0$, 0.87 for $\Delta \mathrm{R} 10,0.88$ for $\Delta \mathrm{R} 20,0.89$ for $\Delta \mathrm{R} 30,0.94$ for $\Delta \mathrm{R} 40$ and $\Delta R 50,0.92$ for $\Delta R 60,0.93$ for $\Delta R 70,0.96$ for $\Delta R 80$ and 0.78 for $\Delta \mathrm{R} 90$.

\section{Discussion}

Predicting caries activity is considered the 'holy grail' of cariology [Ismail et al., 2005]. To date, only subjective methods are available to determine whether a lesion will progress to cavitation or will arrest. It is known that not all early lesions progress to cavitation [Dirks, 1966]. Our study in this population also demonstrated that lesions with rapid changes in QLF parameters like the area, depth and volume of the lesion progressed to cavitation [Ferreira Zandona et al., 2013]. Identifying lesions that are likely to progress to cavitation can have a great impact on how dentistry is practiced and how the caries paradigm 
Fig. 3. ROC predicting progression to cavitation from the final observation for red fluorescence total.

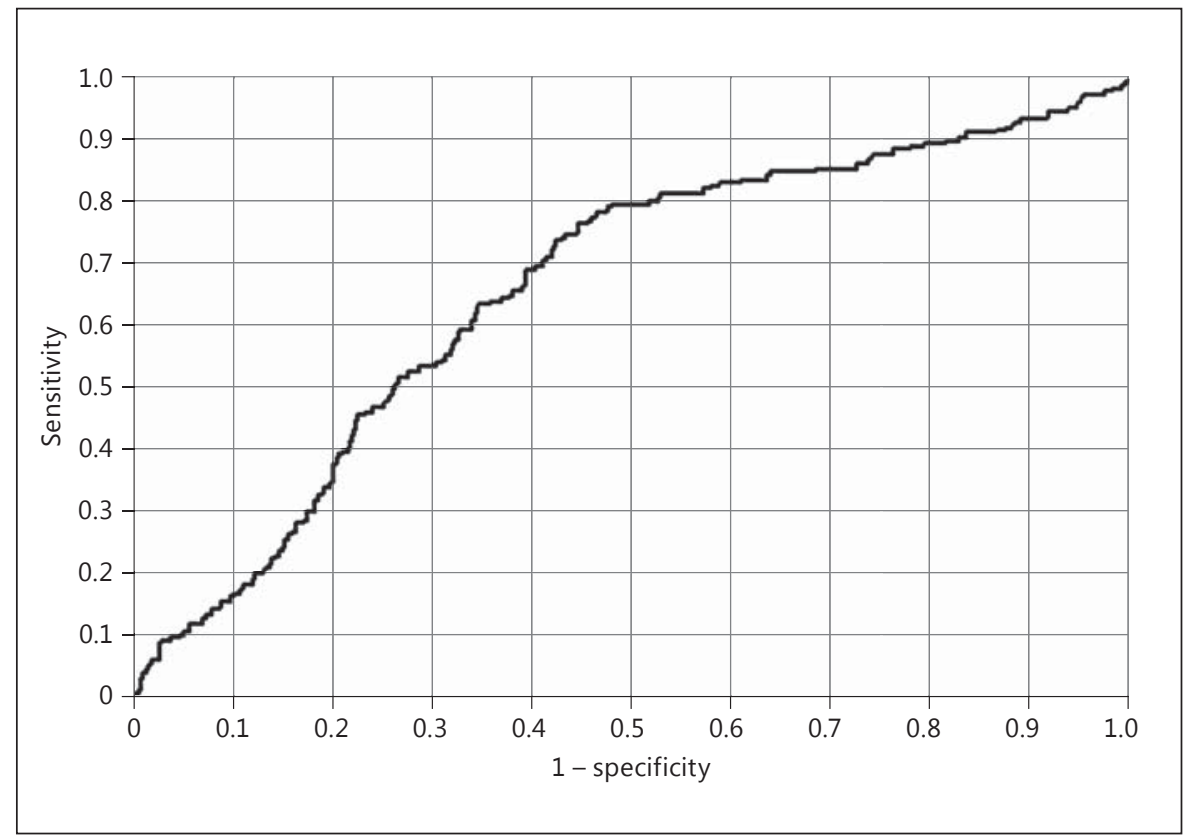

shift towards the nonsurgical management of dental caries can be implemented.

Most studies have focused on the red fluorescence of oral biofilm. The orange-to-red intrinsic fluorescence has been shown to be emanating from the oral biofilm (dental plaque) comprising all the oral bacterial species. It does not come from a single bacterial species [van der Veen et al., 2006]. A red excited fluorescence signal is likely to be derived from metabolic byproducts of oral bacteria in the dental biofilm [Koenig and Schneckenburger, 1994]. Red fluorescence emission seen on QLF images is proposed to be the result of excitation of endogenous porphyrins by the violet-blue light at a wavelength range of $380-500 \mathrm{~nm}$. Fluorescing porphyrins in caries detected to some extent are protoporphyrin IX, coproporphyrin and uroporphyrin [Buchalla et al., 2008]. Red fluorescence from bacteria was shown to be an indicator of dentinal carious lesions [Lennon et al., 2006]. Higher levels of red-to-orange fluorescence can be an indicator of progression of lesions, as demonstrated in our study. Early detection of orange/red fluorescence may serve as a caries indicator to predict caries activity.

Our study focused on the tooth surfaces that were cleaned of visible biofilm before imaging. It showed that at specific thresholds or cutoff levels the orange/red fluorescence from lesions that progressed to cavitation was significantly higher compared to nonprogressive carious lesions. At low thresholds of $\Delta \mathrm{R} 0, \Delta \mathrm{R} 10$ and $\Delta \mathrm{R} 20$ at baseline or the first available visit the orange/red fluorescence was significantly higher for the lesions that progressed to cavitation compared to lesions that did not progress. This indicates that the values of orange/red fluorescence have a potential to identify lesions that are likely to be active, that is, progress towards cavitation. This may be the first nonsubjective means to determine caries activity at a single time point. This can have a significant impact on dental care, ranging from caries risk to specific caries intervention.

Yet, there are several questions that remain to be answered. In our study, at every visit prior to capturing QLF images the teeth were brushed and flossed by study personnel. However, orange/red fluorescence was not only observable in the lesions, but also an apparent indicator of caries activity. What is the origin of this red fluorescence - are these metabolic products within the body of the lesion [Buchalla et al., 2008]? Could we just use the red fluorescence from the biofilm for caries prediction as some studies seem to indicate [Buchalla et al., 2010]?

Within the limitations of the study showing moderate sensitivity, quantification of orange/red fluorescence was able to distinguish carious lesions that progressed to cavitation from lesions that did not progress at a single observation. Though there are several factors involved in the increased contribution of orange/red fluorescence, future studies identifying the microbiological factors causing the orange/red fluorescence and its implication in caries 
activity are indicated. Additionally, studies are needed to understand the role of porphyrins in active and arrested carious lesions.

\section{Acknowledgments}

The study was partially supported by NIH/NIDCR grant RO1DE017890. The funders had no role in the study design, data collection and analysis, decision to publish, or preparation of the manuscript. We thank the faculty and staff at Indiana University and the University of Puerto Rico, who were part of the parent study, in particular the examiners, Drs. Haftstein Eggertsson and Enrique Santiago. We would especially like to thank Dr. Richard L. Gregory, Dr. Domenick Zero, Dr. Masatoshi Ando, Ms. Sue Kelly, Ms. Melissa Mau, Ms. Sharon Gwinn, Ms. Jennifer Tran, Dr. Mahmoud Jallad and the supporting staff at the Oral Health Research Institute, Indiana University School of Dentistry, for all the support given. We thank Dr. Elbert de Josselin Jong for the help related to the QLF software.

\section{Author Contributions}

Grace Felix Gomez, PhD student in Dental Sciences, Indiana University School of Dentistry, carried out the image analysis and wrote the paper, and also contributed to the conception of the article. Mr. George J. Eckert, Biostatistician supervisor, Indiana University School of Medicine, did the statistical analysis, tables and figures, and critically revised the manuscript for accuracy and approved it upon changes. Dr. Andrea Ferreira Zandona was the mentor for this project and the principal investigator of the parent study. She supervised the collection of images and the clinical assessment of the surfaces in the parent study. She facilitated the study design, the input of extensive ideas from conceptualization and data interpretation, as well as critically analyzing the paper and providing final approval upon changes.

\section{Disclosure Statement}

The authors declare no potential conflicts of interest with respect to the authorship and/or publication of this article.

\section{References}

Alammari MR, Smith PW, de Josselin de Jong E, Higham SM: Development of caries indices using quantitative light-induced fluorescence (QLF) in vitro. ICQ 3rd International Conference on Quantitative Light-Induced Fluorescence (QLF), Liverpool, 2010.

Alfano RR, Yao SS: Human teeth with and without dental caries studied by visible luminescent spectroscopy. J Dent Res 1981;60:120122.

Benedict HC: A note on the fluorescence of teeth in ultra-violet rays. Science 1928;67:442.

Bittar DG, Pontes LR, Calvo AF, Novaes TF, Braga MM, Freitas PM, Tabchoury CP, Mendes FM: Is the red fluorescence of dental plaque related to its cariogenicity? J Biomed Opt 2014;19:065004.

Bjelkhagen H, Sundstrom F, Angmar-Mansson B, Ryden H: Early detection of enamel caries by the luminescence excited by visible laser light. Swed Dent J 1982;6:1-7.

Buchalla W: Comparative fluorescence spectroscopy shows differences in noncavitated enamel lesions. Caries Res 2005;39:150-156.

Buchalla W, Attin T, Niedmann Y, Niedmann PD, Lennon AM: Porphyrins are the cause of red fluorescence of carious dentine: verified by gradient reversed-phase HPLC. Caries Res 2008; $42: 223$.

Buchalla W, Lennon AM, Attin T: Fluorescence spectroscopy of dental calculus. J Periodontal Res 2004a;39:327-332.

Buchalla W, Lennon AM, Attin T: Comparative fluorescence spectroscopy of root caries lesions. Eur J Oral Sci 2004b;112:490-496.
Buchalla W, Lennon A, Techert S, Krause J, Becker K, Attin T: Dental biofilm fluorescence may indicate caries risk. Caries Res 2010;44:230.

Coulthwaite L, Pretty IA, Smith PW, Higham SM, Verran J: The microbiological origin of fluorescence observed in plaque on dentures during QLF analysis. Caries Res 2006;40:112116.

de Josselin de Jong E, Sundstrom F, Westerling H, Tranaeus S, ten Bosch JJ, Angmar-Mansson $B$ : A new method for in vivo quantification of changes in initial enamel caries with laser fluorescence. Caries Res 1995;29:2-7.

Dirks OB: Posteruptive changes in dental enamel. J Dent Res 1966;45:503-511.

Ferreira Zandona A, Ando M, Gomez GF, GarciaCorretjer M, Eckert GJ, Santiago E, Katz BP, Zero DT: Longitudinal analyses of early lesions by fluorescence: an observational study. J Dent Res 2013;92:84s-89s.

Ferreira Zandona A, Eggertson H, Wefel J, Barry K, Ofner S, Eckert G: Clinical validation study of QLF at Indiana; in Stookey GK (ed): Early Detection of Dental Caries III: Proceedings of the 6th Indiana Conference. Indiana University School of Dentistry, Indianapolis, 2003, pp 363-373.

Ferreira Zandona A, Santiago E, Eckert G, Fontana M, Ando M, Zero DT: Use of ICDAS combined with quantitative light-induced fluorescence as a caries detection method. Caries Res 2010;44:317-322.

Ferreira Zandona A, Santiago E, Eckert GJ, Katz BP, Pereira de Oliveira S, Capin OR, Mau M, Zero DT: The natural history of dental caries lesions: a 4-year observational study. J Dent Res 2012;91:841-846.
Fontana M, Santiago E, Eckert GJ, Ferreira-Zandona AG: Risk factors of caries progression in a Hispanic school-aged population. J Dent Res 2011;90:1189-1196.

Ismail AI, Banting D, Eggertsson H, Ekstrand K, Ferreira-Zandona A, Longbottom C, Pitts NB, Reich E, Ricketts D, Selwitz R, Sohn W, Topping S, Doughlas GV, Zero D: Rationale and evidence for the international caries detection and assessment system (ICDAS II); in Stookey GK (ed): Proceedings of the 7th Indiana Conference. Indiana University, Indianapolis, 2005, pp 161-221.

Kim YS, Lee ES, Kwon HK, Kim BI: Monitoring the maturation process of a dental microcosm biofilm using the quantitative light-induced fluorescence-digital (QLF-D). J Dent 2014;42: 691-696.

Koenig K, Schneckenburger H: Laser-induced autofluorescence for medical diagnosis. J Fluoresc 1994;4:17-40.

König K, Flemming G, Hibst R: Laser-induced autofluorescence spectroscopy of dental caries. Cell Mol Biol (Noisy-le-Grand, France) 1998;44:1293-1300.

König K, Schneckenburger H, Hibst R: Time-gated in vivo autofluorescence imaging of dental caries. Cell Mol Biol (Noisy-le-Grand, France) 1999;45:233-239.

Lee ES, Kang SM, Ko HY, Kwon HK, Kim BI: Association between the cariogenicity of a dental microcosm biofilm and its red fluorescence detected by quantitative light-induced fluorescence-digital (QLF-D). J Dent 2013;41: 1264-1270. 
Lennon AM, Buchalla W, Brune L, Zimmermann O, Gross U, AttinT: The ability of selected oral microorganisms to emit red fluorescence. Caries Res 2006;40:2-5.

Pitts N: 'ICDAS' - an international system for caries detection and assessment being developed to facilitate caries epidemiology, research and appropriate clinical management. Community Dent Health 2004;21:193-198.

Slimani A, Panayotov I, Levallois B, Cloitre T, Gergely C, Bec N, Larroque C, Tassery H, Cuisinier F: Porphyrin involvement in redshift fluorescence in dentin decay; in Biophotonics: Photonic Solutions for Better Health Care IV: Proceedings of SPIE, 2014. Brussels, 2014, vol 9129.
Stubel H: Die Fluoreszenz tierischer Gewebe in ultraviolettem Licht. Pflugers Arch Physio 1911;142:1-14.

Sundstrom F, Fredriksson K, Montan S, Hafstrom-Bjorkman U, Strom J: Laser-induced fluorescence from sound and carious tooth substance: spectroscopic studies. Swed Dent J 1985;9:71-80.

van der Veen MH, Buchalla W, de Josselin de Jong E: QLFTM technologies: recent advances; in Stookey GK (ed): Proceedings of the 6th Indiana Conference. Indiana University School of Dentistry, Indianapolis, 2003, pp 291-304.

van der Veen MH, Thomas RZ, Huysmans MC, de Soet JJ: Red autofluorescence of dental plaque bacteria. Caries Res 2006;40:542-545.
Volgenant CM, van der Veen MH, de Soet JJ, ten Cate JM: Effect of metalloporphyrins on red autofluorescence from oral bacteria. Eur J Oral Sci 2013;121:156-161.

Waller E, van Daelen CJ, van der Veen MH: Application of QLFTM for Diagnosis and Quality Assessment in Clinical Practice, 2012. Inspektor Research Systems. http://www.inspektor.nl/download/WhitepaperQLF11.pdf (accessed August 31, 2015).

Zandona AF, Zero DT: Diagnostic tools for early caries detection. J Am Dent Assoc 2006;137: 1675-1684; quiz 1730.

Zezell DM, Ribeiro AC, Bachmann L, Gomes AS, Rousseau C, Girkin J: Characterization of natural carious lesions by fluorescence spectroscopy at $405-\mathrm{nm}$ excitation wavelength. Biomed Opt 2007;12:064013. 